\title{
Coordinated STEM/FIB/NanoSIMS Analyses of Presolar Silicates in Comet Dust and Primitive Meteorites
}

\author{
L. Keller ${ }^{1}$, A. Nguyen ${ }^{2}$, Z. Rahman ${ }^{2}$ and S. Messenger ${ }^{1}$ \\ ${ }^{1}$ Robert M. Walker Laboratory for Space Science, ARES, Mail Code KR, NASA Johnson Space \\ Center, Houston, Texas 77058 USA \\ ${ }^{2}$ Jacobs ESCG, NASA Johnson Space Center, Houston, Texas 77058 USA
}

Silicate grains were among the most abundant mineralogical building blocks of our Solar System. These grains were the detritus from earlier generations of stars that have been recycled in the early solar nebula. Rare sub-micrometer survivors of this processing have been identified in meteorites, micrometeorites and interplanetary dust particles (IDPs). These silicate grains are recognized as presolar in origin because of their extremely anomalous isotopic compositions that reflect nucleosynthetic processes in their stellar sources (evolved stars, novae and supernovae). We perform coordinated chemical, mineralogical and isotopic studies of these grains to determine their origins and histories.

We examine the complex mineralogy and petrography of presolar silicates using imaging, diffraction and chemical data obtained from thin sections with the JSC JEOL 2500 field-emission STEM equipped with a Noran thin window energy dispersive x-ray (EDX) spectrometer and a Gatan Tridiem GIF. Quantitative element X-ray maps (spectrum images) are acquired by rastering a $4 \mathrm{~nm}$ incident probe whose dwell time is minimized to avoid beam damage and element diffusion during mapping. Successive image layers are acquired and combined in order to achieve $1 \%$ counting statistics for major elements. The IDP samples are prepared by ultramicrotomy of particles embedded in epoxy or elemental sulfur. After EDX mapping, the sections are subjected to $\mathrm{C}, \mathrm{N}$, and $\mathrm{O}$ isotopic imaging with the JSC NanoSIMS 50L ion microprobe. We prepare sections of some meteorite grains using the JSC FEI Quanta 3D focused ion beam (FIB) instrument. The specimen surface is protected from the FIB milling process by layers of electron beam-deposited $\mathrm{C}$ and $\mathrm{Pt}$ followed by an ion-deposited Pt layer. We also use the FIB to preferentially remove surrounding grains to reduce the background in subsequent NanoSIMS measurements. For mineralogical studies, we again employ the FIB instrument to deposit a protective cap over the grain of interest and then extract the grain and thin it to electron transparency for TEM analysis.

Figure 1 shows an example of a presolar amorphous silicate grain observed in a microtome thin section of an IDP. The grain is an aggregate with four distinct sub-grains in the $\mathrm{Mg} / \mathrm{Si}$ ratio image, all of which contain nanophase inclusions on FeNi metal and FeS. The mineralogical and isotopic properties indicate this aggregate formed as a non-equilibrium condensate in the atmosphere of an asymptotic giant branch star. We also have analyzed presolar silicate grains from meteorites by ion imaging in the NanoSIMS to identify anomalous grains. These analyses are enabled by the FIB techniques described above. We have identified a grain whose $\mathrm{O}$ and $\mathrm{Mg}$ isotopic compositions are consistent with a supernova (SN) source (Fig. 2). Quantitative x-ray mapping shows the grain is stoichiometric enstatite $\left(\mathrm{MgSiO}_{3}\right)$, yet diffraction data and dark-field imaging show that it is amorphous. This grain likely formed as crystalline enstatite and was subsequently rendered amorphous, either in the interstellar medium or in the environment near the site of formation. These studies illustrate the mineralogical diversity of presolar silicates from different stellar sources. 

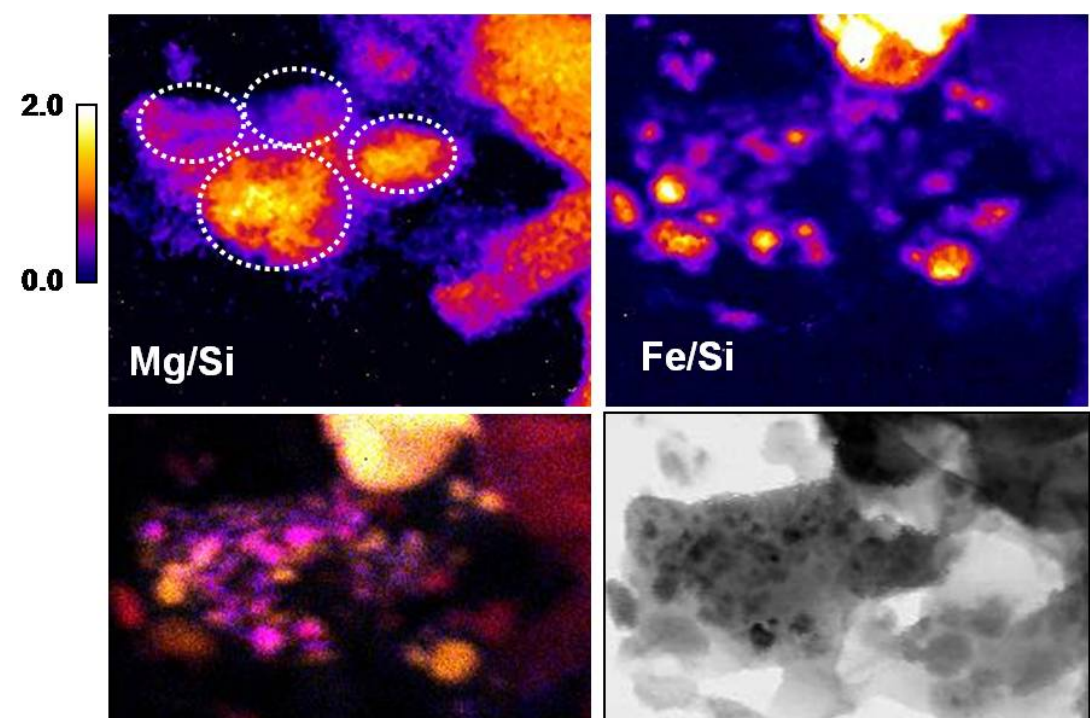

$\mathrm{Fe}+\mathrm{Ni}+\mathrm{S}$

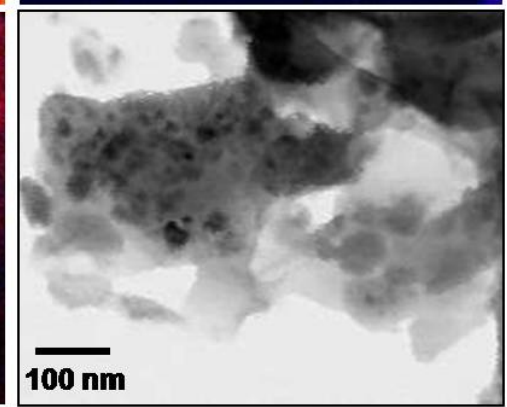

Figure 1. Coordinated analysis of a presolar amorphous silicate grain $\left(\delta^{17} \mathrm{O} \sim 495 \%\right)$. The grain is heterogeneous with several sub-grains apparent (circled) in the quantitative $\mathrm{Mg} / \mathrm{Si}$ atom ratio image. $\mathrm{The} \mathrm{Fe}+\mathrm{Ni}+\mathrm{S}$ map shows a heterogeneous distribution of nanophase metal and sulfide inclusions. Lower right image is a brightfield STEM image of the presolar silicate grain.
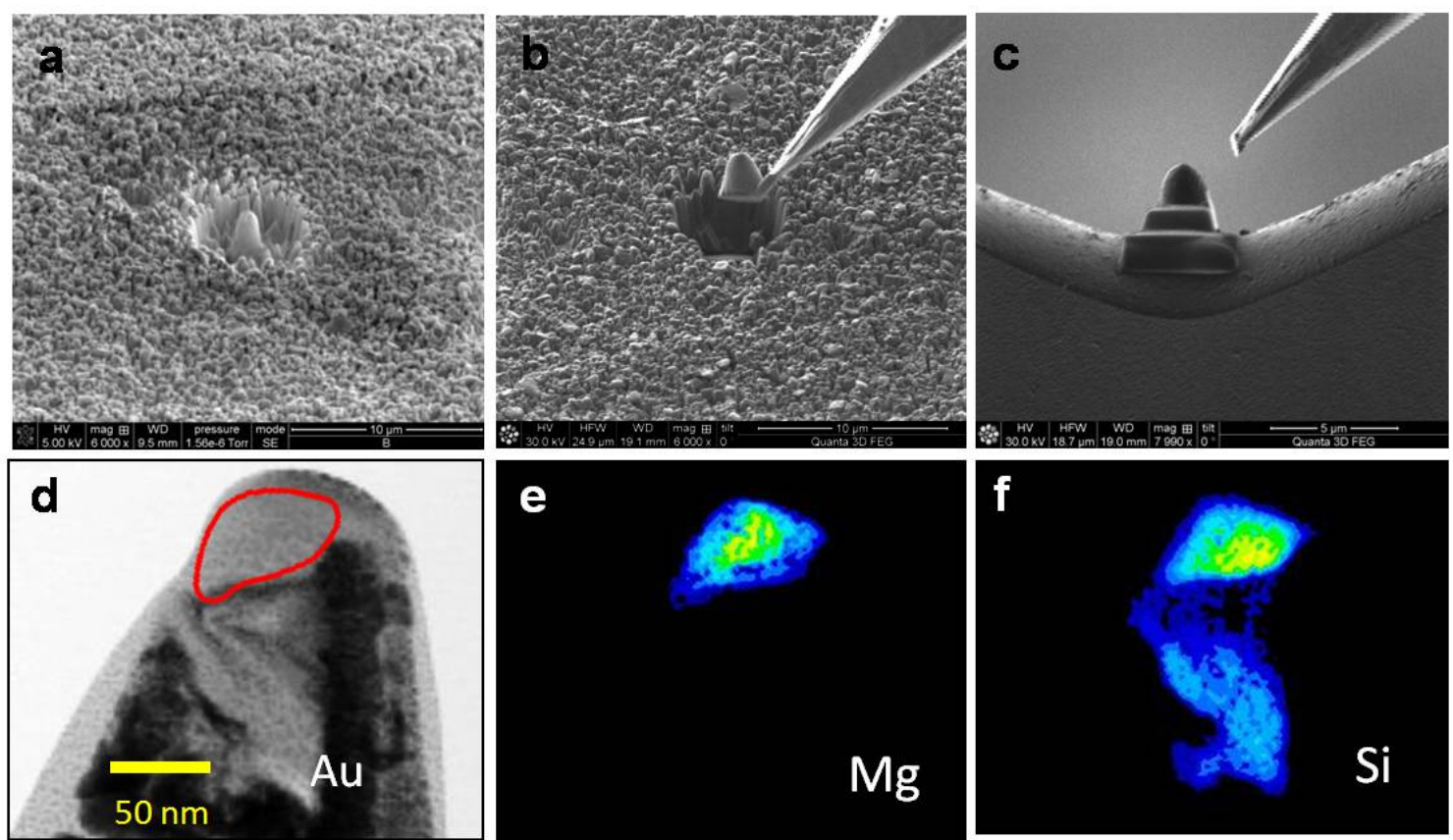

Figure 2. Coordinated analysis of supernova (SN) silicate grain $\left(\delta^{17} \mathrm{O} \sim 1495 \%\right.$, $\delta^{18} \mathrm{O} \sim 2434 \%$, $\delta^{25} \mathrm{Mg} \sim-177 \%$, $\delta^{26} \mathrm{Mg} \sim 357 \%$ ): a) SN grain isolated using FIB, b) FIB lift-out of the SN grain on top of Au substrate, c) Attaching the FIB section to a TEM grid, d) Brightfield TEM image showing amorphous SN grain at tip of section (red circle), e) Mg x-ray map of the FIB section and f) Si x-ray map of the section. 